University of Nebraska - Lincoln

DigitalCommons@University of Nebraska - Lincoln

September 1999

\title{
Comparison of magnetic images using point and thin-film magnetic force microscopy tips
}

Sy_Hwang Liou

University of Nebraska-Lincoln, sliou@unl.edu

Follow this and additional works at: https://digitalcommons.unl.edu/physicsliou

Part of the Physics Commons

Liou, Sy_Hwang, "Comparison of magnetic images using point and thin-film magnetic force microscopy tips" (1999). Si-Hwang Liou Publications. 61.

https://digitalcommons.unl.edu/physicsliou/61

This Article is brought to you for free and open access by the Research Papers in Physics and Astronomy at DigitalCommons@University of Nebraska - Lincoln. It has been accepted for inclusion in Si-Hwang Liou Publications by an authorized administrator of DigitalCommons@University of Nebraska - Lincoln. 


\title{
Comparison of Magnetic Images Using Point and Thin-Film Magnetic Force Microscopy Tips
}

\author{
S. H. Liou \\ Department of Physics and Astronomy, and \\ Center for Materials Research and Analysis \\ University of Nebraska, Lincoln Nebraska 68588-0111
}

\begin{abstract}
The magnetic images of a magnetic reference sample were compared using point and thin-film magnetic force microscopy (MFM) tips. The thin film MFM tip was made by magnetron sputtering of an amorphous metal. The point MFM tip was made by an ion milling process that produces a small magnetic particle on the cantilever. Our results clearly demonstrated that the volume of magnetic material involved in the tip-sample interaction is much reduced in the case of the point tip than in that of the thin film tips. By comparing the magnetic images of a tri-bit pattern on a magnetic reference sample, we observed an improved resolution using a point MFM tip.
\end{abstract}

Index Terms - Magnetic force microscopy, magnetic force microscopy tip, magnetic images of recording disks, resolution of magnetic images.

\section{INTRODUCTION}

Magnetic force microscopy (MFM) images are usually modeled as a monopole or dipole in which the magnetic charges near the tip point is assumed to be constant [1-2]. However, the magnetic interaction is a long-range interaction that includes not only the interaction between the sample and the magnetic material near the tip, but also from the extended area of the tip. This makes it more difficult to interpret MFM images. One of the ways to improve the resolution of MFM images and faith in MFM images is to improve the magnetic tips used, by developing tip coating techniques or by modifying tip-shape [3-6]. In this paper, we compare the magnetic domain images obtained by using a point MFM tip and a thin-film MFM tip. The image obtained from the point tip shows much less effects due to the stray field from the extended area of the tip. The spatial resolution of the MFM images using the point tip is clearly better than that of the thin-film tip.

\section{EXPERIMENT}

The tips used in this experiment were commercial silicon micromachined cantilevers with spring constants of $1-5 \mathrm{~N} / \mathrm{m}$, resonant frequencies of $70-89 \mathrm{kHz}$, and quality factors of about 200 in air. The thin film MFM tips were made by magnetron sputtering of an amorphous metal of $\mathrm{Fe}_{67} \mathrm{Co}_{18} \mathrm{~B}_{14} \mathrm{Si}_{1}(2605 \mathrm{CO})$. The thickness of the coating is 30 nm.

Manuscript received March 5,1999.

Sy-Hwang Liou, 364 Behlen, University of Nebraska; Lincoln, NE 68588-0111, USA, Phone: (402) 472-2405, Fax: (402) 472-2879, E-Mail: sliou@unlinfo.unl.edu.

Research was supported by the Army Research Office under grant number DAAG55-98-1-0014.
The amorphous $2605 \mathrm{CO}$ alloy is a soft magnetic material that has been used as the core of high power transformers. One of the unique features of amorphous alloy is its structure and magnetic isotropy. The domain structure of an amorphous magnetic material is expected to be less sensitive to the shape of the tip than a crystalline magnetic material. The magnetization of the amorphous alloy, 1.75 T, is about two times higher than that of Permalloy. The high magnetization is essential for the improvement of the signal/noise ratio. In general, the smaller tip magnetic moment will improve the spatial resolution and give easy to interpret MFM images, but may demand improved signal-tonoise. The coercivity field of these tips was about $250 \mathrm{~A} / \mathrm{m}$, smaller than the typical stray field of hard disk recording media. We also noted that the magnetic properties of the amorphous $2605 \mathrm{CO}$ alloy were sensitive to preparation conditions [7].

The point MFM tips were prepared as follows: (a) The magnetic thin film tip was mounted on a scanning probe microscope. The photo-resist film was painted on the glass slide. (b) The tip was gradually dipped into the photo-resist film by controlling the force on the tip. The coating size on the tip can be easily controlled by the thickness of the photoresist film, which was about $0.5-1 \mu \mathrm{m}$. (c) The unprotected magnetic film was then removed by ion sputtering performed in a vacuum chamber. (d) The photo-resist on the tip was removed by acetone. As shown in Fig. 1 a MFM cantilever with a magnet size of $500 \mathrm{~nm}$ on the tip is clearly observed.

The sample investigated was a reference sample made from a recording disk typical of those currently found in hard-disk drives. The reference sample has been characterized by P. Rice et al. [8]. One of the advantages of using the reference sample is that the same area can be easily located and compared.

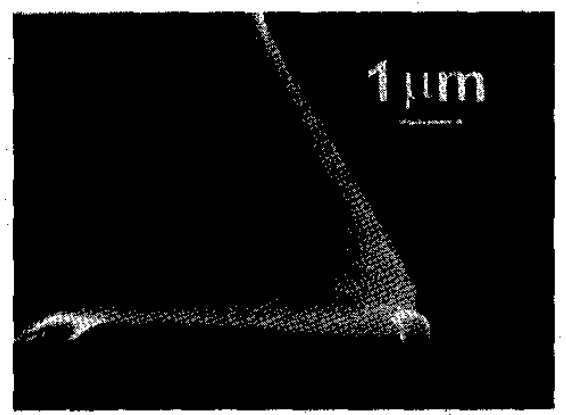

Fig. 1 A MFM cantilever with a magnet size of $500 \mathrm{~nm}$ on the tip. 


\section{RESULTS AND DISCUSSION}

The topographic and magnetic images were taken simultaneously, permitting direct correlation of magnetic profile to the topography of the reference sample.

Topographic data was taken by an atomic force microscope (AFM) in intermittent contact and magnetic data (MFM images) was recorded with the tip at a fixed distance from the surface of the thin-film recording medium [9-10]. In this method, the force gradient between the sample and the sensing tip was measured by the frequency shift of the cantilever. In non-contact scanning force microscopy, such as the MFM mode, the magnetic interaction is a long-range interaction; thus spatial resolution depends on both the dimension of the tip and the distance between the tip and the sample. In this study, the gap (tip-sample distance) was about $25 \mathrm{~nm}$ [11].

We compared the images obtained from the thin film tip and the point tip. Both tips are coated with same magnetic material and show a soft magnetic behavior (i.e. the tip magnetic moment follows the stray field of the sample.) As shown in the top of Fig. 2, these atomic force microscopy images, taken from a reference sample, are from the same area. For a soft MFM tip, the interaction of the stray field from the recording disk with the tip generates a magnetic force derivative that is always attractive (as shown by the dark lines). The bottom of Figs. $2 a$ and $2 b$ shows the MFM images that were obtained from the thin-film tip. The bottom of Fig. $2 \mathrm{c}$ is the MFM image that was obtained from the point tip. The difference between Fig $2 a$ and $2 b$ is that the tip was magnetized in an opposite direction. The magnetization direction of the tip was magnetized perpendicular to the sample surface. Fig $2 a$ and $2 b$ shows the effects of the magnetic interactions from an extended area of the tips. At further distances from the sample, which has a smaller stray field, the tip magnetization does not flip completely. As a result, the tri-bit pattern (indicated by an arrow) was not resolved, as shown in Fig 2a and $2 b$. We observed either one dark line or two dark lines for the tri-bit pattern using a thin film tip that has a different magnetization direction. In contrast, Fig $2 \mathrm{c}$ shows three dark lines for the tri-bit pattern using a point tip. This can be better illustrated by Fig. 3. The insets of Fig. 3 are the schematic of the magnetization of the MFM tip near the tri-bit pattern. It illustrates that the magnetic interactions between the tip and the sample can be complicated, because the magnetic hysteresis in the magnetic force microscopy (MFM) tips causes history-dependent interactions between the tip and the sample.

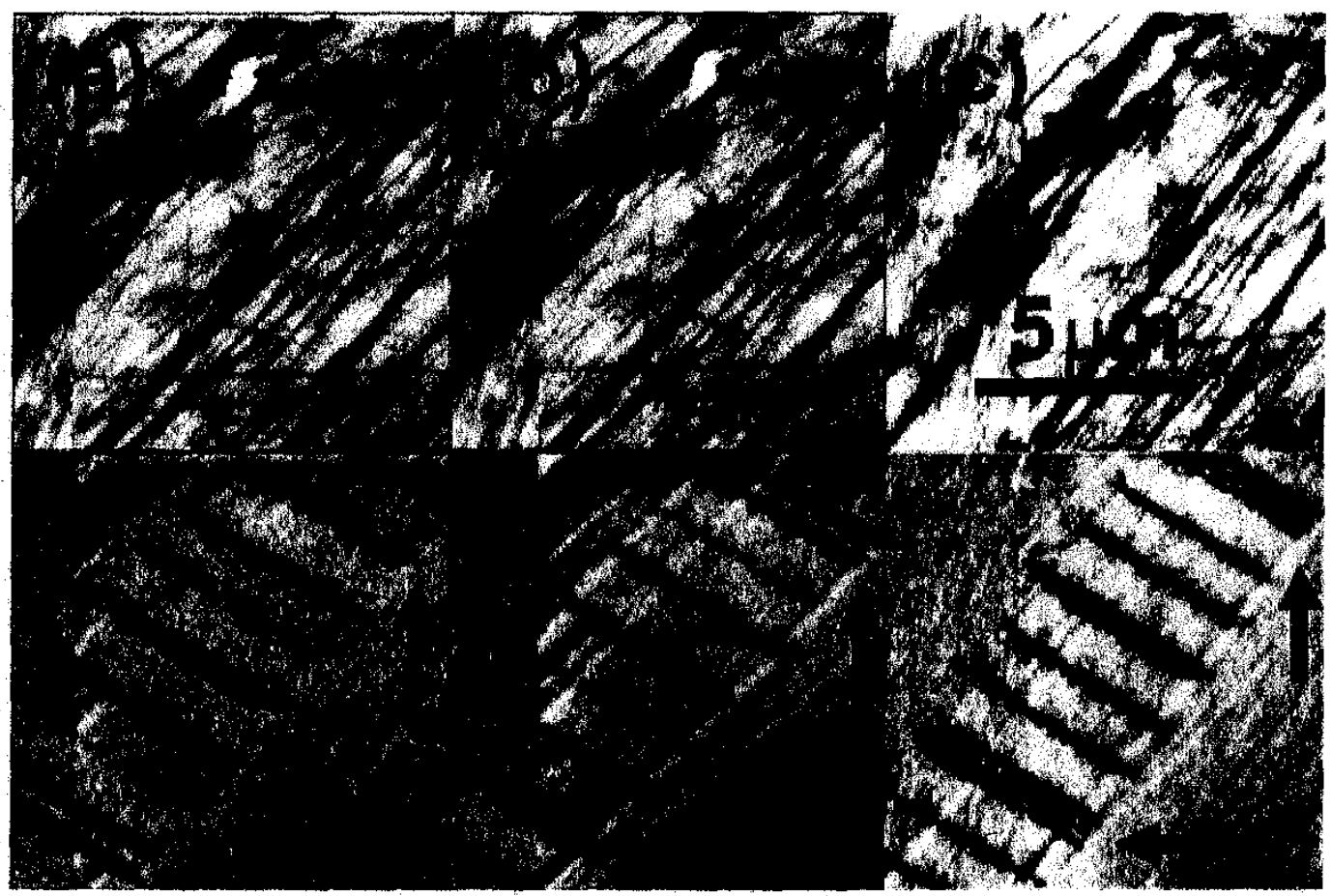

Fig. 2 A comparison of the MFM images with the thin-film tip ( $2 a$ and $2 b$ ) and the point tip ( $2 c$ ). The details explained in the text. 

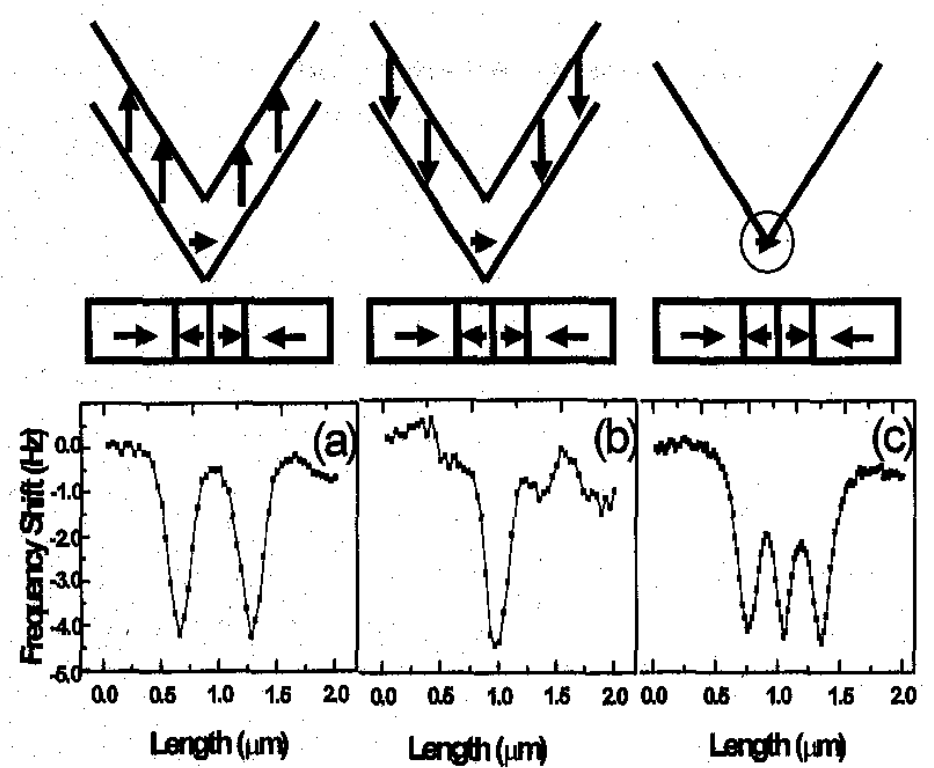

Fig. 3 Frequency shifts on $2 \mu \mathrm{m}$ line traces across a tri-bit pattern on the reference sample with the thin-film tip ( $3 \mathrm{a}$ and $3 \mathrm{~b}$ ) and the point tip ( $3 \mathrm{c}$ ). The scale of the frequency shift at Fig. $3 \mathrm{c}$ is 5 times less than that of Fig. $3 \mathrm{a}$ and $3 \mathrm{~b}$. The inset is the schematic drawing of the tip magnetization. The actual magnetization direction of the tip is not known. Due to the shape anisotropy of the film at tip, It is expected that have a component other than $\mathrm{z}$-direction.

Figs. $3 \mathrm{a}$ and $3 \mathrm{~b}$ are the $2 \mu \mathrm{m}$ trace across the tri-bit area from the thin-film tip. Fig. $3 \mathrm{c}$ is the $2 \mu \mathrm{m}$ trace across the tribit area from the point tip. The traces in Fig $3 a$ and $3 b$ show that the tri-bit patterns were not resolved. There is either two peaks or one peak, depending on the magnetization direction of the tip. It is clearly shown in the insets of Fig. $3 a$ and $3 b$ that the magnetic interaction between the stray field of the sample and the magnetization directions at the side-wall of the tip is the key factor for the difference. In contrast, Fig 3c shows the magnetization of the tip as it follows the stray field of the sample, possessing three peaks, with essentially no effect due to the magnetization interaction from the side-wall of the tip. However, The sensitivity of the point tip is about 5 time less than that of the thin film tips.

This comparison clearly shows that the point tip produced a better-resolved MFM image because it was not affected by the magnetic interactions from the extended area of the tip.

This observation is consistent with a theoretical analysis that showed the difference in the point response as a function of the tip geometry and the tip-to sample spacing [1]. The estimated result from the theoretical analysis shows that the point dipole tip has a resolution of $0.6 \mathrm{r}$ ( $\mathrm{r}$ is the radius of curvature of the apex of the tip), while the finite size tip has a broader response (1.25r) and thus lower resolution.

\section{SUMMARY}

In summary, we have shown better resolution MFM images of a reference sample using a point MFM tip. The tribit pattern with widths less than $200 \mathrm{~nm}$ are well resolved.

\section{ACKNOWLEDGMENT}

The author thanks John Moreland, Paul Rice and Steve Russek at the National Institute of Standards and Technology for discussion and provision of the reference sample that was. used in this study.

\section{REFERENCES}

[1] P. Griatter, H. J. Mamin, and D. Rugar, "Magnetic Force Microscopy," in Scanning Probe Microscopy II, New York: Springer-Verlag, 1991.

[2] Hans J. Hug, B. Stiefel, A. Moser, I. Parashikov, A. Klicznik, D. Lipp, H. -J. Güntherodt, Gabriel Bochi, D. I. Paul and R. C. O'Handley, "Magnetic domain structure in ultrathin $\mathrm{Cu} / \mathrm{Ni} / \mathrm{Cu} / \mathrm{Si}(001)$ films (invited)", J. Appl. Phys. 79, 5609(1996)

[3] P. Gritter, D. Rugar; H. J. Mamin, G. Castillo, S, E. Lambert, C. J. Lin, R. M. Valletta, O. Wolter, T. Bayer, and J. Greschner, "Batch fabricated sensors for magnetic force microscopy", Appl. Phys. Lett., 57, $1820(1990)$

[4] M. Ruhrig, S. Porthun, and J.C. Lodder, "Magnetic force microscopy using electron-beam fabricated tips", Rev. Sci. Instrum. 65, 3224(1994)

[5] P.F. Hopkins, John Moreland, S. S. Malhotra and S. H. Liou, "Superparamagnetic Magnetic Force Microscopy Tips" J, Appl. Phys., 79,6448 (1996).

[6] S. H. Liou, Y. D. Yao, "Development of high coercivity magnetic force microscopy tips", J. Magn. Magn. Mater., 190, 130(1998).

[7] C. Shearwood, A.D. Mattingley, M.R.J. Gibbs, "Growth and pattering of amorphous FeSiBC films", J. Magn. Magn. Mater., 162, 147(1996).

[8] Paul Rice, Stephen E. Russek, J̦ay Hoinville, and Michael H. Kelly, "Optimizing the NIST magnetic imaging reference smaple", IEEE Tran. Magn,, Vol. 33, 4065 (1997).

[9] Q. Zhong, D. Inniss, K. Kjoller, and V. B. Elings, Surf, Sci. Lett., 290, L688 (1993)

[10] Steffen Porthun, Leon Abelmann, and Cock Lodder, "Magnetic force microscopy of thin film media for high density magnetic recording", J. Magn. Magn. Mater., 182, 238(1998).

[11] Sumio Hosaka, Ataushi Kikukawa and Yukio Honda, "High resolution of magnetic force microscope image using a just-on-surface magnetic force microscope", Jpn. J. Appl. Phys., 33, 3779(1994). 